\title{
THE PHYSICAL MOTION OF INTRINSIC SPIN AND THE EXPERIMENTAL IMPLICATION
}

\author{
Gregory L. Light \\ Department of Finance \\ Providence College \\ Providence, Rhode Island 02918, USA
}

\begin{abstract}
We propose a revised Stern-Gerlach experiment of setting up two perpendicular magnetic fields $\mathrm{B}(1)$ and $\mathrm{B}(2)$; we predict that half of the electrons will be observed to align with $\mathrm{B}(1)$ and the other half, $\mathrm{B}(2)$, so that any electron, left (right) - handed, has four spin states, up (down), left (right), down (up), and right (left), in a $720^{\circ}$-cycle, altering the basis for quantum computing.
\end{abstract}

AMS Subject Classification: $81 \mathrm{P} 16,81 \mathrm{P} 10,81 \mathrm{P} 15$

Key Words: Stern-Gerlach experiment re-examined, four spin states, enigma of spin one-half, Dirac spinor

\section{Introduction and Motivation}

Quantum computation has been resting on the basic tenet of the existence of two spin states, up or down, for the electron (cf. e.g., [4], [5]). This paper re-examines this assertion. From Einstein's mass-shell equation

$$
E^{2}=(p c)^{2}+\left(m_{0} c^{2}\right)^{2}
$$

Dirac derived the equation

Received: May 17, 2017

(c) 2017 Academic Publications 


$$
\left(i \hbar \frac{\partial}{\partial t}-i \hbar c \sigma_{i} \nabla\right)\left(\psi_{\text {up }}, \psi_{\text {down }}\right)=m_{0} c^{2}\left(\psi_{\text {up }}, \psi_{\text {down }}\right)
$$

for a free electron, which rendered in Feynman ([3], p. 10-13, with notation adapted to this paper) the following set of differential equations

$$
i \hbar \frac{d}{d t}\left(\begin{array}{c}
\psi_{1}(t) \\
\psi_{2}(t)
\end{array}\right)=-\mu\|\boldsymbol{B}\| \sigma_{z}\left(\begin{array}{c}
\psi_{1}(t) \\
\psi_{2}(t)
\end{array}\right)
$$

for an electron situated in a magnetic field $\mathbf{B}=\left(0,0, B_{z}>0\right)$, with the electron rest mass suppressed to zero. Since the two columns of the Pauli matrix

$$
\sigma_{z} \equiv\left(\begin{array}{cc}
1 & 0 \\
0 & -1
\end{array}\right)
$$

represent two orthogonal vectors that do not correspond to a pair of up and down directions as in a magnetic dipole moment, we thus surmise that the actual wave motion of the electron in the entire process is:

$$
\text { up, left, down, and right, }
$$

in a $720^{\circ}-$ cycle, with (up, left) accounting for $\frac{\hbar}{2}$ in the first $360^{\circ}$ turn and (down, right) accounting for $\frac{\hbar}{2}$ in the second $360^{\circ}$ turn; i.e., there exist four spin states in a $720^{\circ}-$ cycle for all spin $-\frac{1}{2}$ particles. In this way, (up $\perp$ left) or (down $\perp$ right) would correspond to the two orthogonal columns of $\sigma_{z}$. As such, we propose a revised Stern-Gerlach experiment that sets up two perpendicular magnetic fields to test our hypothesis.

An extensive literature research has yielded few results on the subject of intrinsic spin possibly because this construct has become a standard pillar in quantum physics (cf. [2] for an earlier study of this concept, [1] for a study of the spin structure of the nucleon, and [6] for observed 3-dimensional spinning motions). Section 2 below will present our hypothesis in details and Section 3 will conclude with a summary remark.

\section{A Hypothetical Motion of Electron Spins in Euclidean $\mathbb{R}^{3}$}

We begin with the following short-hand notation for easy visualization:

$$
\begin{aligned}
E & \equiv \text { East } \equiv(1,0,0), W \equiv \text { West } \equiv(-1,0,0) \\
N & \equiv \text { North } \equiv(0,1,0), S \equiv \text { South } \equiv(0,-1,0) \\
T & \equiv \text { Top } \equiv(0,0,1), B \equiv \text { Bottom } \equiv(0,0,-1)
\end{aligned}
$$


relative to any frame in question. We hypothesize that any left-handed electron $e_{L}$ has the following spinning motion relative to a laboratory frame (the righthanded electron $e_{R}$ would have the time-reversed spinning motions):

$$
S \rightarrow E \rightarrow N \rightarrow B \rightarrow S
$$

in a $360^{\circ}$ - cycle composed of: (1) a $180^{\circ}$ - rotation along the $(x, y)$ - plane, and $(2)$ a $180^{\circ}$ - rotation along the $(y, z)$ - plane with $e_{L}$ ended upside down.

The angular momenta of $e_{L}$ are thus:

(1) $\frac{\hbar}{2}$ by the motion of $S \rightarrow E \rightarrow N$, a counterclockwise rotation relative to the $z$-axis of $e_{L}$, which is assigned a special label $T_{e_{L}}$ (connoting the Top of $e_{L}$ as bounded to the moving frame of $e_{L}$ ), and

(2) $\frac{\hbar}{2}$ by the motion of $N \rightarrow B \rightarrow S$, a counterclockwise rotation relative to the $x$-axis of $e_{L}$, which is assigned a special label $E_{e_{L}}$ (connoting the East of $e_{L}$ as bounded to the moving frame of $e_{L}$ ), which however has been rotated by the above motion (1) from $E$ to $W$, so that in the laboratory frame motion (2) becomes clockwise while motion (1) remains to be counterclockwise.

Consider an externally imposed magnetic field

$$
\begin{aligned}
\boldsymbol{B} & =\|\boldsymbol{B}\|\left(-\cos \theta_{B}, 0, \sin \theta_{B}\right)(\text { tesla }) \\
0 & \leq \theta_{B} \leq \frac{\pi}{2} .
\end{aligned}
$$

Then motion (1) has wavefunction, with $\omega, t>0$,

$$
C_{1}(t) \equiv \psi_{2}(t)=\sin \theta_{B} e^{i \omega t},
$$

and motion (2) has wavefunction

$$
C_{2}(t) \equiv \psi_{1}(t)=\cos \theta_{B} e^{-i \omega t} .
$$

The above $\left(C_{1}(t), C_{2}(t)\right)$ thus serve as the basis directions for the Pauli matrix

$$
\begin{aligned}
\sigma_{z} & \equiv\left(\begin{array}{cc}
1 & 0 \\
0 & -1
\end{array}\right), \text { with } \\
i \hbar \frac{d}{d t}\left(\begin{array}{c}
\psi_{1}(t) \\
\psi_{2}(t)
\end{array}\right) & =-\frac{\mu}{2}\|\boldsymbol{B}\| \sigma_{z}\left(\begin{array}{c}
\psi_{1}(t) \\
\psi_{2}(t)
\end{array}\right) ;
\end{aligned}
$$

here in Equation $(2), \mu \equiv$ electron magnetic dipole moment $\approx-9.28 \times 10^{-24}$ joule/tesla is divided by 2 in contrast to the whole factor $\mu$ as contained in the original Equation (1) by Feynman for we are hypothesizing that Equation (1) 
is based on a $720^{\circ}$ - cycle and Equation (2) is based on a $360^{\circ}$ - cycle; also classically

$$
\begin{aligned}
\mu \times \mathbf{B} & =\tau \equiv \text { torque, and } \\
|\tau| & =|\mu| \cdot|\mathbf{B}| \cdot \sin \theta
\end{aligned}
$$

is the energy needed to displace $\mu$ from $\boldsymbol{B}$ by $\theta$. At $\theta_{B}=0$, the laboratory is to observe $E_{e_{L}}$ at $W$; at $\theta_{B}=\frac{\pi}{2}$, the laboratory is to observe $T_{e_{L}}$ at $T$. In general,

$$
\begin{aligned}
\operatorname{Prob}\left(E_{e_{L}} \text { at } W\right) & =\cos ^{2} \theta_{B} \text { and } \\
\operatorname{Prob}\left(T_{e_{L}} \text { at } T\right) & =\sin ^{2} \theta_{B} ;
\end{aligned}
$$

in either event the observed angular momentum is $\hbar / 2$ due to $180^{\circ}=360^{\circ} / 2$.

We thus propose a Stern-Gerlach experiment of setting up two magnetic fields,

$$
\begin{aligned}
& \boldsymbol{B}_{1}=\|\boldsymbol{B}\|(0,0,1) \text { and } \\
& \boldsymbol{B}_{2}=\|\boldsymbol{B}\|(-1,0,0) ;
\end{aligned}
$$

we predict that half of the electrons will be observed to align with $\boldsymbol{B}_{1}$ and the other half, $\boldsymbol{B}_{2}$.

After the above first $360^{\circ}$ rotation, the second $360^{\circ}$ rotation is to have $E_{e_{L}}$ observed at $E$ or $T_{e_{L}}$ observed at $B$. Setting $\theta_{B}=\frac{\pi}{2}$, one then has, over the entire $720^{\circ}$ - cycle,

$$
\begin{aligned}
\operatorname{Prob}\left(T_{e_{L}} \text { at } T\right) & \lesssim \frac{1}{2} \lesssim \operatorname{Prob}\left(T_{e_{L}} \text { at } B\right), \quad \text { or } \\
\operatorname{Prob}(U p) & \lesssim \frac{1}{2} \lesssim \operatorname{Prob}(\operatorname{Down}),
\end{aligned}
$$

where the " $\lesssim$ " sign reflects the fine-structure, with spin-up carrying an angular momentum $\hbar-\delta$ and spin-down, $\hbar+\delta$, so that they sum to $2 \hbar$ over the $720^{\circ}-$ cycle. One then would recover Equation (1),

$$
\begin{aligned}
& i \hbar \frac{d}{d t}\left(\begin{array}{c}
\psi_{2}(t), \omega t \in[2 \pi, 4 \pi] \\
\psi_{2}(t), \omega t \in[0,2 \pi]
\end{array}\right) \\
= & -\mu\|\boldsymbol{B}\|\left(\begin{array}{cc}
1 & 0 \\
0 & -1
\end{array}\right)\left(\begin{array}{l}
\psi_{2}(t), \text { cycle } 2 \\
\psi_{2}(t), \text { cycle } 1
\end{array}\right),
\end{aligned}
$$

where the first column of $\sigma_{z}$, being orthogonal to the second column, would have to serve as an abstract label (as in heads/tails in Bernoulli trials of a coin 
tossing) for the physical observation of $T_{e_{L}}$ at $B \equiv(0,0,-1)$. This $180^{\circ}$ separation between $T \equiv(0,0,1)$ and $B \equiv(0,0,-1)$ for the up-down interpretation of the quantum two states is necessary for a correspondence with magnetic dipole moment, but one finds vagueness in the literature on the definition of "up" and "down", whether referring to abstract probability outcomes or a physical separation of $180^{\circ}$, and our above analysis clarifies this ambiguity.

\section{Summary Note}

The significance of our above hypothesis is that if it is experimentally verified, then any electron, $e_{L} / e_{R}$, has four spin states, up/down, left/right, down/up, and right/left (instead of the established two states, up and down), in a $720^{\circ}-$ cycle, altering the foundation of quantum mechanics as well as computing. We thus suggest physics laboratories re-conduct Stern-Gerlach experiment as specified herein at the earliest possible times.

\section{References}

[1] C.A. Aidala, S.D. Bass, D. Hasch, G.K. Mallot, The spin structure of the nucleon, Rev. Mod. Phys., 85 (2013), 655-691.

[2] H.C. Corben, Spin in classical and quantum theory, Phys. Rev., 121 (1961), 1833-1839.

[3] R.P. Feynman, R.B. Leighton, M. Sands, The Feynman Lectures on Physics, Volume III: Quantum Mechanics, Addison-Wesley, Reading (1965).

[4] E. Kapit, Universal two-qubit interactions, measurement, and cooling for quantum simulation and computing, Phys. Rev. A, 92 (2015), \# 012302 (8 pages).

[5] A.R.P. Rau, Mapping two-qubit operators onto projective geometries, Phys. Rev. A, 79 (2009), \# 042323 (4 pages).

[6] R.S. Van Dyck, Jr., P.B. Schwinberg, H.G. Dehmelt, Electron magnetic moment from geonium spectra: Early experiments and background concepts, Phys. Rev. D, 34 (1986), 722-737. 
